\title{
Urine color as an indicator of urine concentration in pregnant and lactating women
}

\author{
Amy L. McKenzie ${ }^{1}$ (D) Colleen X. Muñoz ${ }^{2} \cdot$ Lindsay A. Ellis $^{3} \cdot$ Erica T. Perrier $^{4}$. \\ Isabelle Guelinckx ${ }^{4} \cdot$ Alexis Klein $^{4} \cdot$ Stavros A. Kavouras $^{5} \cdot$ Lawrence E. Armstrong $^{1}$
}

Received: 22 June 2015 / Accepted: 12 October 2015 / Published online: 16 November 2015

(C) The Author(s) 2015. This article is published with open access at Springerlink.com

\begin{abstract}
Aim Urine concentration measured via osmolality $\left(U_{\mathrm{OSM}}\right)$ and specific gravity $\left(U_{\mathrm{SG}}\right)$ reflects the adequacy of daily fluid intake, which has important relationships to health in pregnant (PREG) and lactating (LACT) women. Urine color $\left(U_{\mathrm{COL}}\right)$ may be a practical, surrogate marker for whole-body hydration status.

Purpose To determine whether $U_{\mathrm{COL}}$ was a valid measure of urine concentration in PREG and LACT, and pairmatched non-pregnant, non-lactating control women (CON). Methods Eighteen PREG/LACT (age $31 \pm 1$ years, prepregnancy BMI $24.3 \pm 5.9 \mathrm{~kg} \mathrm{~m}^{-2}$ ) and eighteen CON (age $29 \pm 4$ years, BMI $24.1 \pm 3.7 \mathrm{~kg} \mathrm{~m}^{-2}$ ) collected 24-h and single-urine samples on specified daily voids at five time points $(15 \pm 2,26 \pm 1$, and $37 \pm 1$ weeks gestation, $3 \pm 1$ and $9 \pm 1$ weeks postpartum during lactation; CON visits were separated by similar time intervals) for measurement of 24-h $U_{\mathrm{OSM}}, U_{\mathrm{SG}}$, and $U_{\mathrm{COL}}$ and single-sample $U_{\mathrm{OSM}}$ and $U_{\mathrm{COL}}$.
\end{abstract}

Amy L. McKenzie

amylmckenzie@gmail.com

1 Human Performance Laboratory, Department of Kinesiology, University of Connecticut, 2095 Hillside Road, U1110, Storrs, CT 06269-1110, USA

2 Department of Health Sciences and Nursing, University of Hartford, 200 Bloomfield Ave, West Hartford, CT 06117, USA

3 Centre for Heart Lung and Vascular Health, University of British Columbia, 1147 Research Road, Kelowna, BC V1V 1V7, Canada

4 Danone Research, RD 128, 91767 Palaiseau, France

5 Human Performance Laboratory, Department of Health, Human Performance and Recreation, University of Arkansas, 155 Stadium Drive, HPER 321, Fayetteville, AR 72701, USA
Results Twenty-four-hour $U_{\mathrm{COL}}$ was significantly correlated with 24-h $U_{\mathrm{OSM}}(r=0.6085-0.8390, P<0.0001)$ and 24-h $U_{\mathrm{SG}}(r=0.6213-0.8985, P<0.0001)$ in all groups. A 24-h $U_{\mathrm{COL}} \geq 4$ (AUC $\left.=0.6848-0.9513, P<0.05\right)$ and single-sample $U_{\mathrm{COL}} \geq 4$ (AUC $=0.9094-0.9216, P<0.0001$ ) indicated 24-h $U_{\mathrm{OSM}} \geq 500 \mathrm{mOsm} \mathrm{kg}{ }^{-1}$ (representing inadequate fluid intake) in PREG, LACT, and CON.

Conclusions Urine color was a valid marker of urine concentration in all groups. Thus, PREG, LACT, and CON can utilize $U_{\mathrm{COL}}$ to monitor their daily fluid balance. Women who present with a $U_{\mathrm{COL}} \geq 4$ likely have a $U_{\mathrm{OSM}} \geq 500 \mathrm{mOsm} \mathrm{kg}^{-1}$ and should increase fluid consumption to improve overall hydration status.

Keywords Fluid intake $\cdot$ Hydration status $\cdot$ Biomarker . Urine color $\cdot$ Pregnant women $\cdot$ Lactating women

\section{Introduction}

Water is an essential nutrient [1] and plays a vital role in metabolism by maintaining cellular shape, supporting cellular functions, and serving as a transport medium for nutrients and wastes [2]. Water is especially important to women who are pregnant or nursing. During gestation, plasma volume expands [3], and amniotic fluid protects the developing child [4]. An average woman gains about $11 \mathrm{~kg}$ of body mass during pregnancy, and a large portion of this gain (approximately 7-8 L) is due to water retention [5]. During the postpartum period, nursing mothers experience an increased water loss via breast milk, representing approximately $700 \mathrm{ml}$ per day at 8 weeks postpartum $[6,7]$. These increased physiological needs for water pose a challenge to the neuroendocrine mechanisms that regulate fluid-electrolyte balance and suggest evolving water requirements from conception to lactation. 
Despite the importance of water to both the mother and developing child, research on the regulation of total body water balance, daily water needs, and biomarkers of hydration in pregnant and lactating women is scarce. Even less is known about how maternal hydration may impact either mother or fetus during pregnancy or into early life. Consequently, the dietary reference intakes of water during pregnancy in both Europe and the USA (2.3 and 3.0 $\mathrm{L} \mathrm{day}^{-1}$, respectively) are based only upon the increased amount of water needed to offset the increased caloric intake during pregnancy (i.e., $300 \mathrm{ml} \mathrm{day}{ }^{-1}$ for $300 \mathrm{kcal} \mathrm{day}^{-1}$ ) [8, 9]. During lactation, adequate intake of total water increases to 2.7 [8] and 3.8 [9] $\mathrm{L} \mathrm{day}^{-1}$ in Europe and the USA, respectively, to offset water lost in breast milk.

While dietary reference intakes provide guidelines for adequate intake for the population [8,9], individual water needs vary greatly depending upon personal water intake via the diet, and personal water losses through physical activity, environmental, and other factors. Among the various sources of daily water loss, urine output has been identified as one way to assess hydration in the individual. Urine volume and concentration represent the end result of intake and loss, after accounting for differences in sweat loss and dietary solute load. Manz et al. [10] suggested that 24-h urine concentration reflects the kidney's self-regulation of fluid volume deficit or excess, and provides an indication of the sum of all water gains and losses in healthy individuals. Other recent publications [11, 12] support this concept with data showing that, in healthy men and non-pregnant women, urinary hydration biomarkers including urine osmolality $\left(U_{\mathrm{OSM}}\right)$ and specific gravity $\left(U_{\mathrm{SG}}\right)$ represent inter- and intraindividual differences of daily fluid intake in high- versus low-volume consumers. While useful in a clinical or laboratory setting, $U_{\mathrm{OSM}}$ is not readily available to the general population. However, urine color $\left(U_{\mathrm{COL}}\right)$, a tool that requires little cost, laboratory instrumentation, and expertise, may be valuable as a surrogate hydration marker for daily monitoring of urine concentration. While $U_{\mathrm{COL}}$ has been demonstrated to track changes in daily hydration habits in the general adult population [13-15], no study has assessed its utility or validity in pregnant or lactating women. During pregnancy, serum osmolality is reduced approximately 8-10 mOsm kg ${ }^{-1}$, changing the point of isotonicity between urine and blood at the kidney, potentially impacting free water clearance and $U_{\mathrm{OSM}}$ and thus potentially interrupting the previously reported relationship between $U_{\text {OSM }}$ and $U_{\mathrm{COL}}$ in non-pregnant women. Additionally, some research reports increased void frequency [16] and increased 24-h urine volume with lower 24-h $U_{\mathrm{OSM}}$ in pregnant women [17], but decreased void frequency [16] and decreased 24-h urine volume with higher 24-h $U_{\mathrm{OSM}}$ in lactating women [18]. Given that increased 24-h urine volume is associated with lighter urine color and lower osmolality in non-pregnant, non-lactating women [14], this suggests the relationship between $U_{\mathrm{COL}}$ and $U_{\mathrm{OSM}}$ may be skewed in, or between, pregnant and lactating women compared to control women and warrants further investigation.

Thus, the purpose of this investigation was to determine whether $U_{\mathrm{COL}}$ was a valid measure of urine concentration in pregnant and lactating women, as well as pair-matched non-pregnant and non-lactating controls. The validity of urine color was evaluated in two ways: first, by determining the relationships between 24-h $U_{\mathrm{COL}}$ and biomarkers of urine concentration, specifically 24-h $U_{\mathrm{OSM}}$ and 24-h $U_{\mathrm{SG}}$, utilizing a Spearman's rank-order correlation, and second, by determining the diagnostic accuracy of $U_{\mathrm{COL}}$ (24-h and single samples, separately), to accurately identify $U_{\mathrm{OSM}} \geq 500 \mathrm{mOsm} \mathrm{kg}^{-1}$, utilizing receiver operating characteristic curve analysis, sensitivity, and specificity. Maintaining $U_{\mathrm{OSM}}$ under this cutoff has been associated with ensuring adequate daily fluid intake and urine output for the reduction in risk for urolithiasis or decline in kidney function, as well as avoidance of high plasma vasopressin concentrations often linked to disease states [19].

\section{Methods}

\section{Subjects}

Twenty pregnant women and 18 non-pregnant, control women of similar age, height, and weight participated in this study. Pregnant women enrolled in the study prior to 16-week gestation and reported to the laboratory five times over approximately 8 months. All pregnant women enrolled in the study were breastfeeding during the two postpartum visits. Two pregnant participants were removed from data analysis: one who withdrew from the study and one who developed a gestational condition affecting fluid balance. Non-pregnant, non-lactating control women reported to the laboratory at intervals similar to the pregnant/lactating women and were taking a combination drug oral contraceptive; samples were collected from control women only during the early follicular phase of their menstrual cycle (placebo phase of their pill pack) to limit any effect of exogenous estrogen on osmotically influenced components of fluid balance [20] and to ensure data collection occurred at the same time during their cycle at each visit. Exclusion criteria included use of tobacco products, participation in exercise $>7 \mathrm{~h}$ per week, the presence of a health condition (e.g., type 2 diabetes, polycystic ovarian syndrome) or prescription of a medication that would alter fluid balance, or the development of a gestational condition that would alter fluid balance. The University's Institutional Review Board approved this study, and thus, all study procedures were performed in accordance with ethical standards specified by the Declaration of Helsinki. 


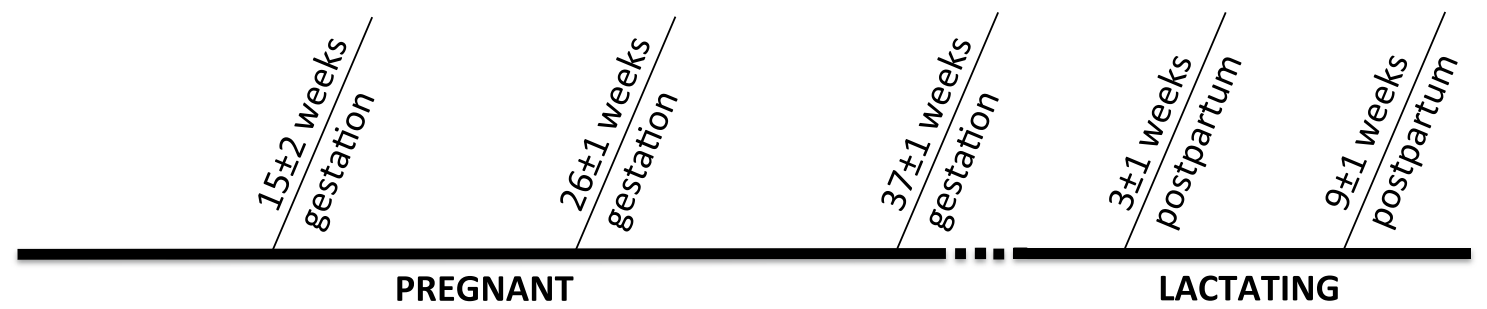

CONTROL

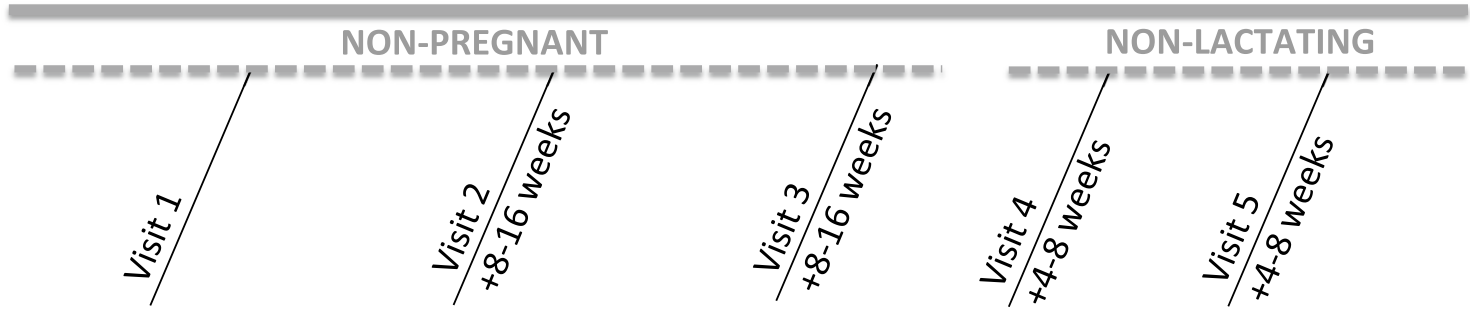

Fig. 1 Experimental time line of visits and data collection. Participants collected urine at five time points across approximately 8 months. For pregnant and lactating women, respectively, time indicates their gestational age relative to their last menstrual period and time relative to delivery. For control women, time indicates the time passed since the former visit. Pregnant and lactating women are the

\section{Experimental design}

Data were collected from pregnant participants at the end of the first ( $15 \pm 2$ weeks gestation), second ( $26 \pm 1$ weeks gestation), and third (37 \pm 1 weeks gestation) trimesters and at $3 \pm 1$ and $9 \pm 1$ weeks postpartum during lactation. Non-pregnant, non-lactating control women were pair-matched on the basis of age, height, and body mass (compared to pre-pregnancy body mass in pregnant participants) and reported for testing at similar intervals to the pregnant/lactating participants (Fig. 1). Participants selfreported age, parity, and pre-pregnancy body mass. Prior to each visit, women were instructed to eat and drink as they normally would. At each visit, height and body mass were recorded. Height was measured with a stadiometer, and weight was measured to the nearest $0.01 \mathrm{~kg}$ (Health $\mathrm{O}$ Meter, Model 349KLX, Alsip, IL). Body mass index was calculated as $\mathrm{kg} \mathrm{m}^{-2}$. Researchers provided each participant with clean urine containers, and the women collected all urine for $24 \mathrm{~h}$ before each visit in a large container along with specific single, spot urine samples as follows: participants collected a single sample of their last urine void of the day and consumed $200 \mathrm{ml}$ of water before going to sleep, collected a single sample of any overnight urine voids (thus, the number of overnight urine samples varied between women), and collected a single sample of their first urine void of the day resulting in a minimum of two single, spot samples of urine for each woman. After measurements were taken on the single samples, these single samples were pooled with the remaining collection to form same participants, and non-pregnant and non-lactating women are the same participants, but at different time points. These four groups were utilized in correlational analyses with visits $1-3$ and visits 4-5 pooled. For diagnostic accuracy analyses, data from non-pregnant and non-lactating women (visits 1-5) were pooled

a complete 24-h urine sample; the same measurements were taken on the full 24-h urine sample.

Urine osmolality, specific gravity, and color were measured for each urine sample. Urine osmolality was determined by freezing point depression (Advanced Instruments Inc., Model 3320, Norwood, MI; CV $=0.3 \%$ ) in duplicate, and $U_{\mathrm{SG}}$ was determined by manual refractometry (Reichert Technologies, Model TS-400, Depew NY). Urine color was determined by a single researcher in a well lit room by placing the sample in a clear container and on a white background next to a previously published chart [21]. The researcher recorded the number of the chart color that most closely matched the urine sample; if the color of the urine sample appeared to fall between two colors on the chart, the researcher chose the darker of the two colors for consistency in assessment technique.

\section{Statistical analysis}

Differences between groups in demographic characteristics were assessed using independent samples $t$ tests at the end of the first trimester $(15 \pm 2$ weeks gestation, visit 1$)$ to compare pregnant (PREG) to non-pregnant (NP) women. Correlations between 24-h $U_{\mathrm{COL}}$ and $U_{\mathrm{SG}}$ and $U_{\mathrm{OSM}}$ were evaluated in PREG (visits 1-3 pooled), NP (visits 1-3 pooled), lactating women (LACT; visits $4-5$ pooled), and non-lactating women (NL; visits 4-5 pooled) with Spearman's rank-order correlations. For diagnostic accuracy analyses, NP and NL were pooled into one group of control women $(\mathrm{CON})$. The diagnostic accuracy of $U_{\mathrm{COL}}$ in 
Table 1 Participant demographics and anthropometrics

\begin{tabular}{lllll}
\hline & $\begin{array}{l}\text { Pregnant/lactating } \\
\text { women }\end{array}$ & $\begin{array}{l}\text { Control } \\
\text { women }\end{array}$ & $t$ & $P$ \\
\hline$n$ & 18 & 18 & \\
Parity (\%) & & & \\
$\quad$ Nulliparous & 28 & 89 & & \\
Primiparous & 55 & 11 & & \\
Multiparous & 17 & 0 & & \\
Age (years) & $31 \pm 3$ & $29 \pm 4$ & 1.382 & 0.176 \\
Height (m) & $1.66 \pm 0.07$ & $1.64 \pm 0.08$ & 0.752 & 0.458 \\
Body mass (kg) & $69.58 \pm 18.57$ & $64.83 \pm 13.84$ & 0.870 & 0.390 \\
Self-reported pre-pregnancy & $66.89 \pm 19.24$ & $64.70 \pm 14.28$ & 0.368 & 0.715 \\
$\quad$ body mass (kg) & & & & \\
Pre-pregnancy BMI (kg m ${ }^{-2}$ ) & $24.3 \pm 5.9$ & $24.1 \pm 3.7$ & 0.134 & 0.895 \\
\hline
\end{tabular}

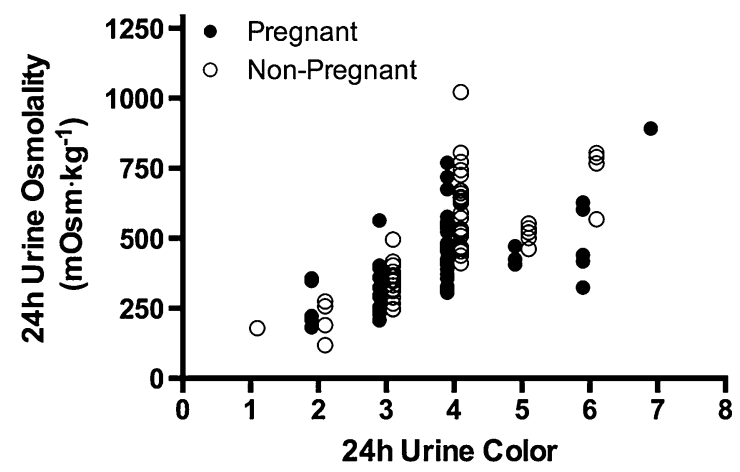

Fig. 2 Relationships of 24-h $U_{\mathrm{COL}}$ to 24-h $U_{\mathrm{OSM}}$ during pregnancy and lactation. Individual data points represent 24-h $U_{\mathrm{COL}}$ and 24-h $U_{\text {OSM }}$ during PREG (visits $1-3$ pooled, $r=0.6085, P<0.0001$ ),

24-h and single samples was assessed in PREG, LACT, and CON using receiver operating characteristic (ROC) curve analysis to determine the ideal $U_{\mathrm{COL}}$ criterion value to identify $U_{\mathrm{OSM}} \geq 500 \mathrm{mOsm} \mathrm{kg} \mathrm{kg}^{-1}[8,19]$. Repeated measures in ROC curve analyses were not accounted for to allow a more conservative analysis [22]. Differences in area under the curve (AUC) were evaluated using methods previously described $[23,24]$ and corrected with a Bonferroni adjustment to account for multiple comparisons. Follow-up calculations of sensitivity and specificity utilizing the criterion value identified by the ROC curve analysis were performed. Level of significance was set a priori at $P<0.05$.

\section{Results}

\section{Demographics and correlations}

At the end of the first trimester, pregnant women were similar to their matched controls in age, height, body mass, self-reported pre-pregnancy body mass, and pre-pregnancy BMI (Table $1 ; P>0.05$ ). Twenty-four-hour $U_{\mathrm{COL}}$

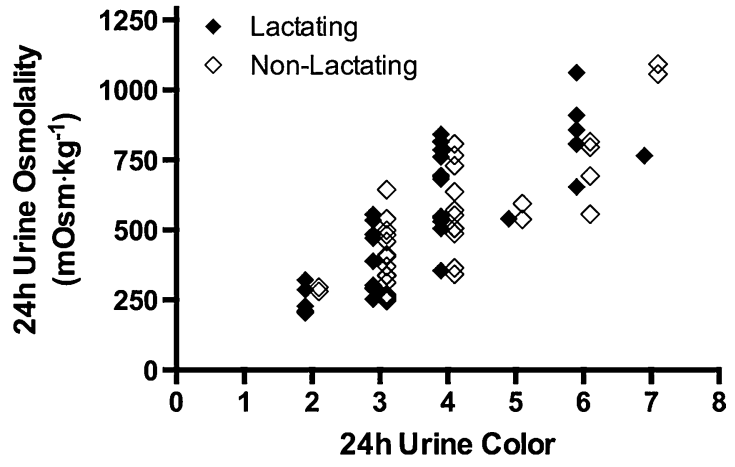

NP (visits $1-3$ pooled, $r=0.7826, P<0.0001$ ), LACT (visits 4-5 pooled, $r=0.8390, P<0.0001$ ), and NL (visits $4-5$ pooled, $r=0.7736, P<0.0001)$

was significantly correlated with 24-h $U_{\mathrm{OSM}}(r=0.61-$ $0.84, P<0.0001$; Fig. 2) and 24-h $U_{\mathrm{SG}}(r=0.62-0.90$, $P<0.0001$; Fig. 3) in all groups.

\section{Diagnostic accuracy of 24-h urine color}

Receiver operating characteristic curve analysis revealed that 24-h $U_{\mathrm{COL}}$ was a useful diagnostic tool to identify 24-h $U_{\mathrm{OSM}} \geq 500 \mathrm{mOsm} \mathrm{kg}^{-1}$ in PREG, LACT, and CON $($ AUC $=0.685-0.951, P<0.05$; Table 2). Area under the curve was significantly different between PREG and LACT $(P=0.002)$ as well as PREG and CON $(P=0.007)$. However, despite significant differences in AUC, all AUC were statistically significant indicating that $24-\mathrm{h} U_{\mathrm{COL}}$ is an accurate indicator of 24-h $U_{\mathrm{OSM}} \geq 500 \mathrm{mOsm} \mathrm{kg}{ }^{-1}$ for all groups, and ROC curve analyses identified the same criterion value with the highest sensitivity and specificity in all groups. A 24-h $U_{\mathrm{COL}}$ criterion value of 4 or higher correctly identified 24-h $U_{\mathrm{OSM}} \geq 500 \mathrm{mOsm} \mathrm{kg}^{-1}$ in more than $90 \%$ of the cases in PREG, LACT, and CON (Table 2; Fig. 4); 24-h $U_{\mathrm{COL}} \geq 4$ and $\geq 5$ demonstrated a trade off between higher sensitivity and higher specificity, respectively, in all groups. 


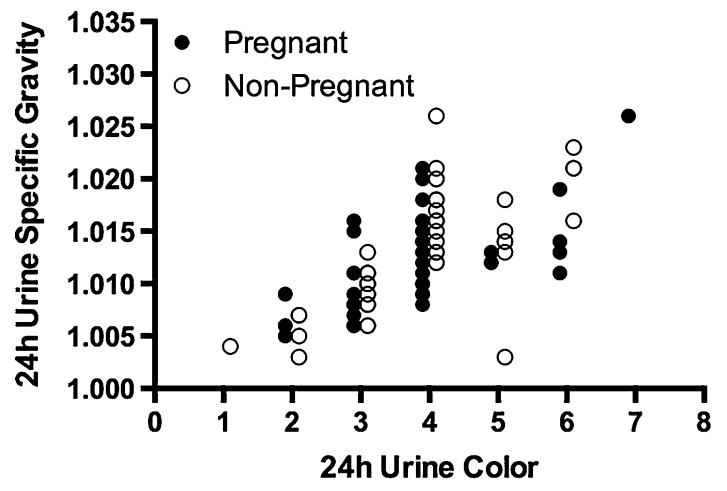

Fig. 3 Relationships of 24-h $U_{\mathrm{COL}}$ to $24-\mathrm{h} U_{\mathrm{SG}}$ during pregnancy and lactation. Individual data points represent 24-h $U_{\mathrm{COL}}$ and 24-h $U_{\mathrm{SG}}$ during PREG (visits $1-3$ pooled, $r=0.6213, P<0.0001$ ), NP (vis-

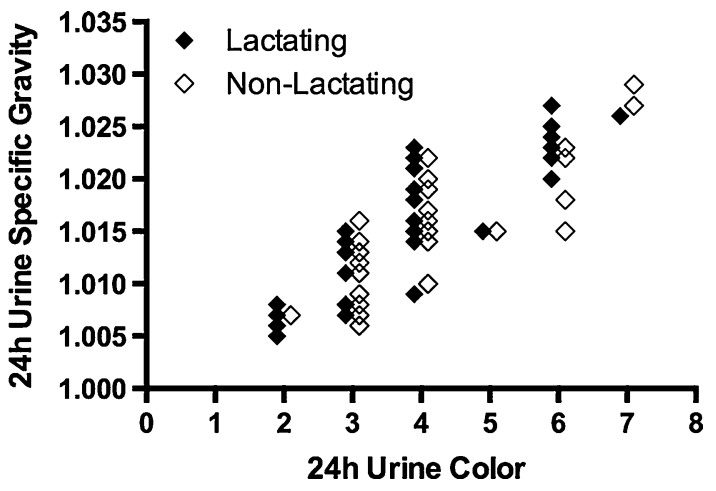

its $1-3$ pooled, $r=0.7416, P<0.0001$ ), LACT (visits $1-3$ pooled, $r=0.8985, P<0.0001$ ), and NL (visits $1-3$ pooled, $r=0.8039$, $P<0.0001)$
Table 2 Receiver operating characteristic curve analysis of $U_{\mathrm{COL}}$ to identify $U_{\mathrm{OSM}} \geq 500 \mathrm{mOsm} \mathrm{kg}^{-1}$

\begin{tabular}{lllllll}
\hline Group & AUC & SE & $P$ & $\begin{array}{l}\text { Criterion } \\
\text { value }\end{array}$ & $\begin{array}{l}\text { Sensitivity } \\
(\%)\end{array}$ & $\begin{array}{l}\text { Specificity } \\
(\%)\end{array}$ \\
\hline 24-h $U_{\text {COL }}$ & & & & & & \\
Pregnant women & 0.6848 & 0.0774 & 0.0463 & 4 & 92 & 91 \\
Lactating women & 0.9513 & 0.0355 & $<0.0001$ & 4 & 91 & 80 \\
Control women & 0.9109 & 0.0310 & $<0.0001$ & 4 & 96 & 70 \\
Single-sample $U_{\text {COL }}$ & & & & & 98 & 78 \\
Pregnant women & 0.9193 & 0.0190 & $<0.0001$ & 4 & 90 & 88 \\
Lactating women & 0.9216 & 0.0244 & $<0.0001$ & 4 & 86 & \\
Control women & 0.9094 & 0.0205 & $<0.0001$ & 4 & & \\
\hline
\end{tabular}

\section{Diagnostic accuracy of urine color in single samples}

Urine color of single samples also was a useful diagnostic tool in PREG, LACT, and CON to identify $U_{\text {OSM }} \geq 500 \mathrm{mOsm} \mathrm{kg} \mathrm{kg}^{-1}$ based on ROC curve analysis (AUC $=0.909-0.922, P<0.0001$; Table 2). Area under the curve was not different between any of the groups $(P>0.05)$. Similar to the 24 -h sample ROC curve analysis, single-sample $U_{\mathrm{COL}}$ criterion values of $\geq 4$ and $\geq 5$ were identified in all groups and represented a trade off between higher sensitivity $\left(U_{\mathrm{COL}} \geq 4\right)$ and higher specificity $\left(U_{\mathrm{COL}} \geq 5\right)$. A single-sample $U_{\mathrm{COL}}$ criterion value of 4 or higher correctly identified $U_{\mathrm{OSM}} \geq 500 \mathrm{mOsm} \mathrm{kg}^{-1}$ in $\geq 86 \%$ of the cases in all groups (Table 2; Fig. 5).

\section{Discussion}

There is no clear consensus on what represents an optimal 24-h urine concentration, either for the general population or specifically for pregnant and lactating women. Approximately $800 \mathrm{mOsm} \mathrm{kg} \mathrm{kg}^{-1}$ has been proposed and used as an upper limit for euhydration [25, 26]. However, 500 mOsm $\mathrm{kg}^{-1}$ has been suggested as a threshold for "appropriate" [27] or "optimal" [19] hydration, supported by observed associations between low 24-h fluid intake, low 24-h urine volume, and/or high 24-h urine concentration and the development of kidney stones [28], urinary tract infection [29], hyperglycemia [30], chronic kidney disease [31], and rate of decline in estimated glomerular filtration rate [32, 33]. Given the heightened importance of water balance in pregnancy, it appears prudent to leave a reasonable margin between a target 24-h urine osmolality and the upper limit for euhydration, and thus, $500 \mathrm{mOsm} \mathrm{kg} \mathrm{kg}^{-1}$ was deemed appropriate. This value is also supported as a desirable 24-h urine osmolality in the EFSA opinion on dietary reference values for water [8].

We adopted a $U_{\text {OSM }}$ under $500 \mathrm{mOsm} \mathrm{kg}^{-1}$ as a goal for pregnant and lactating women to optimize body water balance [8] and avoid potential negative health consequences [19]. However, the technical requirements of measuring $U_{\text {OSM }}$ (i.e., instrumentation, expertise) preclude its use during daily activities and emphasize the need for simple, noninvasive hydration assessment techniques such as $U_{\mathrm{COL}}$ [34-36]. Figures 2 and 3 demonstrate that 24-h $U_{\mathrm{COL}}$ was significantly correlated with $U_{\mathrm{SG}}$ and $U_{\mathrm{OSM}}$ in pregnant and lactating women. While a range of $U_{\mathrm{SG}}$ and $U_{\mathrm{OSM}}$ is associated with each $U_{\mathrm{COL}}$ for pregnant and lactating women, 

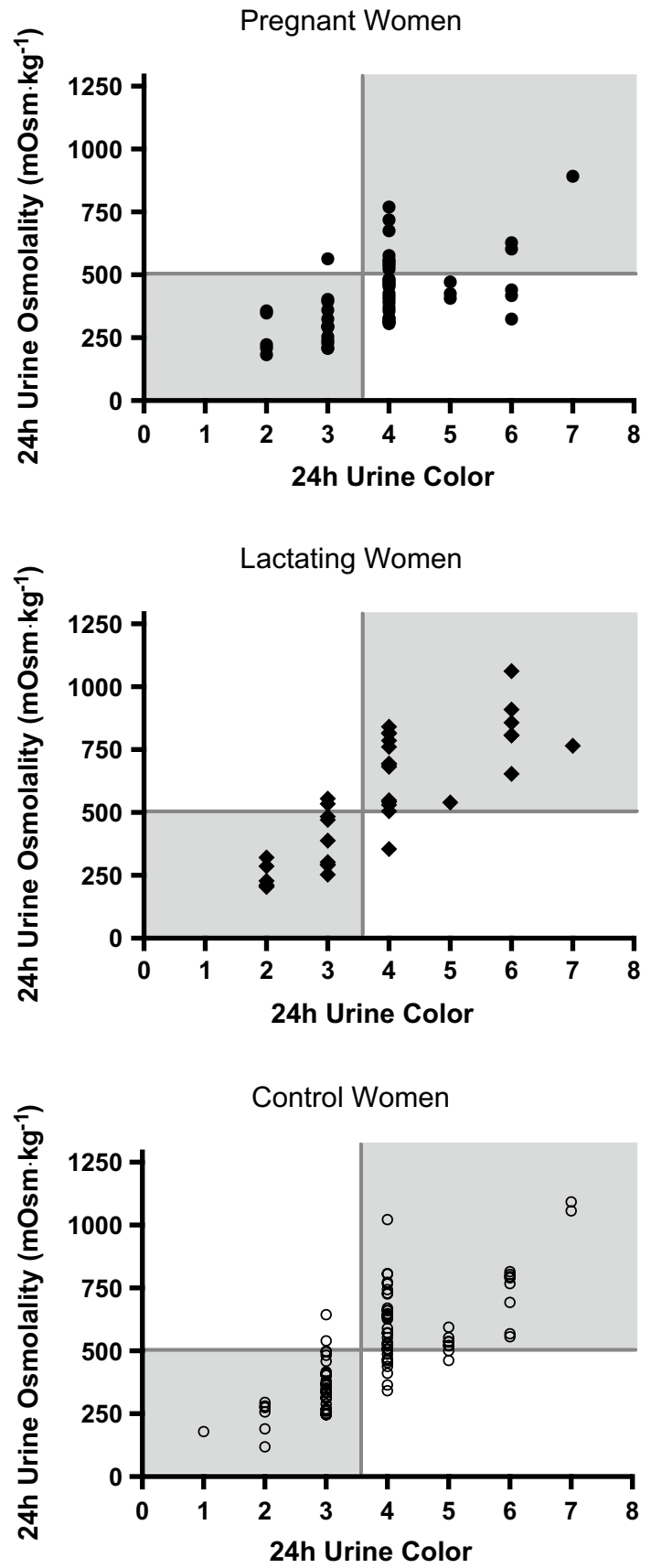

Fig. 4 Contingency plots for 24-h $U_{\mathrm{COL}}$ and 24-h $U_{\mathrm{OSM}}$. Vertical line represents the $U_{\mathrm{COL}} \geq 4$ criterion value determined by the ROC curve analysis to identify $U_{\mathrm{OSM}} \geq 500 \mathrm{mOsm} \mathrm{kg}^{-1}$ (horizontal line)

the degree of variation is similar to relationships demonstrated in other subject populations, including non-pregnant women [14], men [37], the elderly [38, 39], and children [40] where correlation coefficients have ranged from 0.48 to 0.93 . This variation may be explained by the fact that urine color and urine osmolality are generated by two different mechanisms. Urine color is produced by the concentration of urochome in the urine, which is generated by

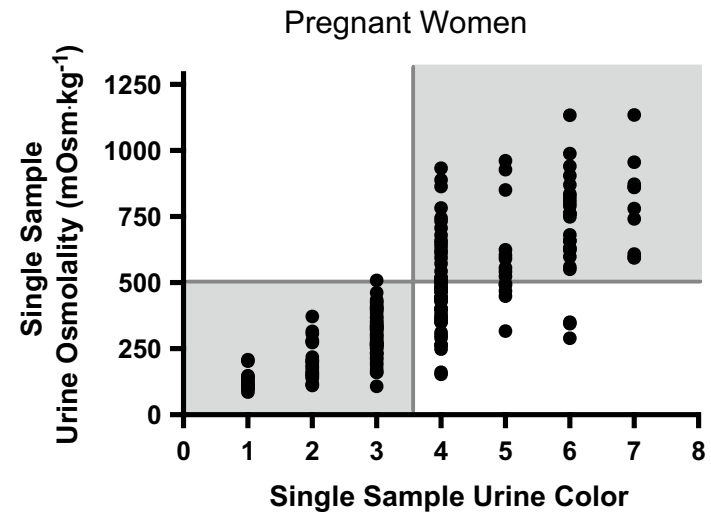

Lactating Women

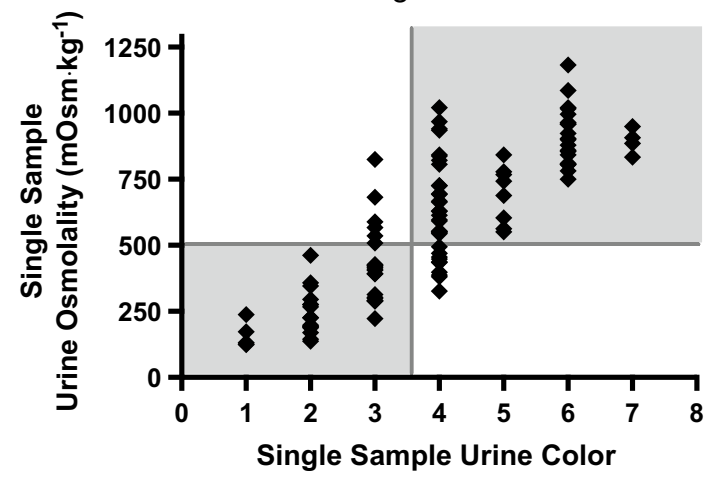

Control Women

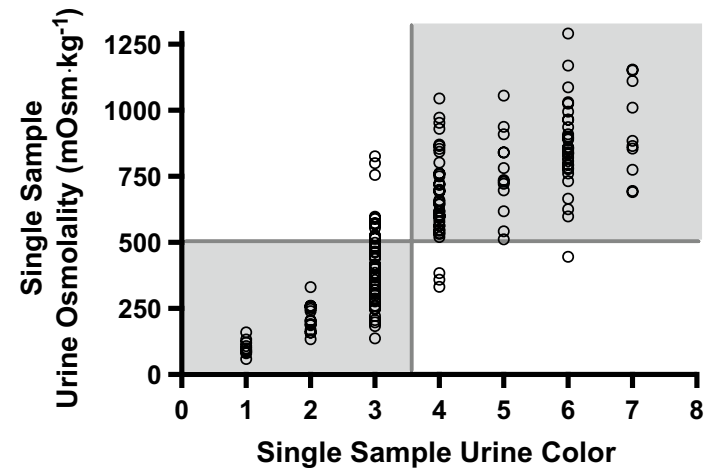

Fig. 5 Contingency plots for $U_{\mathrm{COL}}$ and $U_{\mathrm{OSM}}$ in single samples. Vertical line represents the $U_{\mathrm{COL}} \geq 4$ criterion value determined by the ROC curve analysis to identify $U_{\mathrm{OSM}} \geq 500 \mathrm{mOsm} \mathrm{kg}^{-1}$ (horizontal line)

metabolic processes and independent of diet [41, 42]; urine osmolality is determined by the concentration of solute (e.g., $\mathrm{Na}^{+}, \mathrm{K}^{+}, \mathrm{Cl}^{-}$, urea) in the urine [43]. Despite variation between measures of urine concentration and urine pigmentation, the two remain significantly correlated and adequate means of assessing hydration status in multiple populations.

Further, ROC curve analyses (Table 2) indicated that $U_{\mathrm{COL}}$ provided adequate diagnostic accuracy to allow 
women to successfully monitor $U_{\mathrm{COL}}$ in order to maintain a urine concentration below $500 \mathrm{mOsm} \mathrm{kg} \mathrm{kg}^{-1}$. Because a $U_{\mathrm{COL}} \geq 4$ was associated with high sensitivity (i.e., a high true positive rate), a negative test (i.e., a $U_{\mathrm{COL}}$ of 1,2 , or 3 ) largely ruled-out the possibility of $U_{\mathrm{OSM}}$ being greater than $500 \mathrm{mOsm} \mathrm{kg}{ }^{-1}$. Thus, when a $U_{\mathrm{COL}} \geq 4$ is observed, $U_{\mathrm{OSM}}$ is likely $\geq 500 \mathrm{mOsm} \mathrm{kg}{ }^{-1}$ and warrants increased fluid consumption (assuming no change in solute load) to reduce urine concentration below $500 \mathrm{mOsm} \mathrm{kg} \mathrm{kg}^{-1}$. A visual seemingly equal split of single-sample $U_{\mathrm{OSM}}$ above and below $500 \mathrm{mOsm} \mathrm{kg}^{-1}$ at a $U_{\mathrm{COL}}$ of 4 in pregnant women is present (Figs. 4, 5); this is statistically evident in the trade off between sensitivity and specificity at that criterion value (Table 2). In order to capture as many true positives as possible, the criterion value will favor a higher sensitivity than specificity; thus, a $U_{\mathrm{COL}}$ of 4 emerges as the criterion value to identify $U_{\mathrm{OSM}} \geq 500 \mathrm{mOsm} \mathrm{kg}{ }^{-1}$ with the greatest sensitivity. A lesser AUC in ROC curve analyses for 24-h urine samples in pregnant women statistically represents this variability (Fig. 4; Table 2). While the AUC for $24-\mathrm{h} U_{\mathrm{COL}}$ in pregnant women was significantly different from the other groups, the AUC was still statistically significant, and the criterion value was the same as the criterion value identified in the other groups. This implies that the clinical use of 24-h $U_{\mathrm{COL}}$ in pregnant women to determine whether 24-h $U_{\mathrm{OSM}}$ is $\geq 500 \mathrm{mOsm} \mathrm{kg}^{-1}$ is still valid, but less diagnostically accurate compared to other groups. Future research should evaluate potential sources of this variation.

Monitoring and optimizing water intake during pregnancy and lactation is important, given the inherent physiological challenges to water balance. Increased daily water intake supports expanded total body water, tissue development during gestation, and water loss due to emesis, if experienced. Increased water intake also offsets the water secreted in breast milk. In addition to optimizing body water balance, 24-h water intake impacts fetal and maternal health. For example, acute oral fluid intake of 1-2 L increases amniotic fluid volume in women with both normal and low amniotic fluid volumes [44], but little is known about the effects of habitual maternal fluid intake on development of the child in utero or during the neonatal period. In studies involving hypotonic plasma volume expansion, plasma osmolality decreased for both mother and fetus [45], demonstrating the exchange of fluids between compartments. The change in plasma osmolality and concomitant change in plasma vasopressin has been noted in both acute water loading and 12-h fluid deprivation in pregnant women. Together with the newfound association between high vasopressin and development of preeclampsia [46], these data suggest that adequate water intake during pregnancy might be important for the health of the mother and the fetus. Given the importance of the gestational period and early life on future health outcomes, further research into maternal hydration and health is of fundamental importance. The present investigation may serve as a foundation for future research to identify practical means of monitoring hydration status and enacting behavioral change to promote adequate fluid intake during pregnancy and lactation.

In summary, the present investigation demonstrates that pregnant or lactating women, in addition to nonpregnant and non-lactating women, can use $U_{\mathrm{COL}}$ as a practical indicator of $U_{\mathrm{OSM}}$, and thus, as a reference for whether fluid intake has been adequate. Women who present with $U_{\mathrm{COL}}<4$ likely have a $U_{\mathrm{OSM}}<500 \mathrm{mOsm} \mathrm{kg}{ }^{-1}$, suggesting that their fluid intake is adequate to compensate for daily losses and maintain a healthy urine output. Women who present with $U_{\mathrm{COL}} \geq 4$ likely have a $U_{\mathrm{OSM}} \geq 500 \mathrm{mOsm} \mathrm{kg} \mathrm{kg}^{-1}$ and should increase fluid consumption to reduce urine concentration, thereby reducing the risk of potential health complications [19, 28-33].

Acknowledgment This study was funded by a research grant from Danone Research.

\section{Compliance with ethical standards}

Conflict of interest On behalf of all authors, the corresponding author states that there is no conflict of interest.

Open Access This article is distributed under the terms of the Creative Commons Attribution 4.0 International License (http://creativecommons.org/licenses/by/4.0/), which permits unrestricted use, distribution, and reproduction in any medium, provided you give appropriate credit to the original author(s) and the source, provide a link to the Creative Commons license, and indicate if changes were made.

\section{References}

1. Jéquier E, Constant F (2010) Water as an essential nutrient: the physiological basis of hydration. Eur J Clin Nutr 64:115-123. doi:10.1038/ejen.2009.111

2. Häussinger D (1996) The role of cellular hydration in the regulation of cell function. Biochem J 313(Pt 3):697-710

3. Hytten FE, Paintin DB (1963) Increase in plasma volume during normal pregnancy. J Obstet Gynaecol 70:402-407

4. Goodlin RC, Anderson JC, Gallagher TF (1983) Relationship between amniotic fluid volume and maternal plasma volume expansion. Am J Obstet Gynecol 146:505-511

5. Hytten FE, Thomson AM, Taggart N (1966) Total body water in normal pregnancy. J Obstet Gynaecol 73:553-561

6. Bauer J, Gerss J (2011) Longitudinal analysis of macronutrients and minerals in human milk produced by mothers of preterm infants. Clin Nutr 30:215-220. doi:10.1016/j.clnu.2010.08.003

7. Neville MC, Keller R, Seacat J et al (1988) Studies in human lactation: milk volumes in lactating women during the onset of lactation and full lactation. Am J Clin Nutr 48:1375-1386

8. European Food Safety Authority (2010) Scientific opinion on dietary reference values for water. EFSA J 8:1459. doi:10.2903/j. efsa.2010.1459

9. Institute of Medicine (US) Panel on Dietary Reference Intakes for Electrolytes, Water (2005) DRI, dietary Reference Intakes 
for water, potassium, sodium, chloride, and sulfate. Institute of Medicine of the National Academies, Washington, DC

10. Manz F, Wentz A, Sichert-Hellert W (2002) The most essential nutrient: defining the adequate intake of water. J Pediatr 141:587-592. doi:10.1067/mpd.2002.128031

11. Perrier E, Vergne S, Klein A et al (2013) Hydration biomarkers in free-living adults with different levels of habitual fluid consumption. Br J Nutr 109:1678-1687. doi:10.1017/ S0007114512003601

12. Johnson EC, Muñoz CX, Le Bellego L et al (2015) Markers of the hydration process during fluid volume modification in women with habitual high or low daily fluid intakes. Eur J Appl Physiol 115:1067-1074. doi:10.1007/s00421-014-3088-2

13. Armstrong LE, Pumerantz AC, Fiala KA et al (2010) Human hydration indices: acute and longitudinal reference values. Int $\mathrm{J}$ Sport Nutr Exerc Metab 20:145-153

14. Armstrong LE, Johnson EC, Muñoz CX et al (2012) Hydration biomarkers and dietary fluid consumption of women. J Acad Nutr Diet 112:1056-1061. doi:10.1016/j.jand.2012.03.036

15. Perrier E, Demazières A, Girard N et al (2013) Circadian variation and responsiveness of hydration biomarkers to changes in daily water intake. Eur J Appl Physiol 113:2143-2151. doi:10.1007/s00421-013-2649-0

16. Hong PL, Leong M, Seltzer V (1988) Uroflowmetric observations in pregnancy. Neurourol Urodyn 7:61-70

17. Parboosingh J, Doig A (1973) Studies of nocturia in normal pregnancy. J Obstet Gynaecol 80:888-895

18. van Buul EJ, Steegers EA, Jongsma HW et al (1995) Haematological and biochemical profile of uncomplicated pregnancy in nulliparous women; a longitudinal study. Neth J Med 46:73-85

19. Perrier ET, Buendia-Jimenez I, Vecchio M et al (2015) Twentyfour-hour urine osmolality as a physiological index of adequate water intake. Dis Markers 2015:1-8. doi:10.1155/2015/231063

20. Stachenfeld NS, Silva C, Keefe DL et al (1999) Effects of oral contraceptives on body fluid regulation. J Appl Physiol 87:1016-1025

21. Armstrong LE, Maresh CM, Castellani JW et al (1994) Urinary indices of hydration status. Int J Sport Nutr 4:265-279

22. Liu H, Wu T (2003) Estimating the area under a receiver operating characteristic (ROC) curve for repeated measures design. J Stat Softw 12:1-18

23. Hanley JA, McNeil BJ (1982) The meaning and use of the area under a receiver operating characteristic (ROC) curve. Radiology 143:29-36. doi:10.1148/radiology.143.1.7063747

24. Hanley JA, McNeil BJ (1983) A method of comparing the areas under receiver operating characteristic curves derived from the same cases. Radiology 148:839-843. doi:10.1148/ radiology.148.3.6878708

25. Manz F, Wentz A (2003) 24-h hydration status: parameters, epidemiology and recommendations. Eur J Clin Nutr 57(Suppl 2):S10-S18. doi:10.1038/sj.ejen.1601896

26. Bar-David Y, Urkin J, Kozminsky E (2005) The effect of voluntary dehydration on cognitive functions of elementary school children. Acta Paediatr 94:1667-1673. doi:10.1080/08035250500254670

27. Bar-David Y, Urkin J, Landau D et al (2009) Voluntary dehydration among elementary school children residing in a hot arid environment. J Hum Nutr Diet 22:455-460. doi:10.1111/j.1365-277X.2009.00960.x

28. Curhan GC, Willett WC, Knight EL, Stampfer MJ (2004) Dietary factors and the risk of incident kidney stones in younger women: Nurses' Health Study II. Arch Intern Med 164:885-891. doi:10.1001/archinte.164.8.885

29. Pitt M (1989) Fluid intake and urinary tract infection. Nurs Times 85:36-38

30. Roussel R, Fezeu L, Bouby N et al (2011) Low water intake and risk for new-onset hyperglycemia. Diabetes Care 34:2551-2554. doi: $10.2337 / \mathrm{dc} 11-0652$

31. Strippoli GFM, Craig JC, Rochtchina E et al (2011) Fluid and nutrient intake and risk of chronic kidney disease. Nephrology (Carlton) 16:326-334. doi:10.1111/j.1440-1797.2010.01415.x

32. Clark WF, Sontrop JM, Macnab JJ et al (2011) Urine volume and change in estimated GFR in a community-based cohort study. Clin J Am Soc Nephrol 6:2634-2641. doi:10.2215/ CJN.01990211

33. Sontrop JM, Dixon SN, Garg AX et al (2013) Association between water intake, chronic kidney disease, and cardiovascular disease: a cross-sectional analysis of NHANES data. Am J Nephrol 37:434-442. doi:10.1159/000350377

34. Armstrong LE (2005) Hydration assessment techniques. Nutr Rev 63:40-54. doi:10.1301/nr.2005.jun.S40-S54

35. Fernández-Elías VE, Martínez-Abellán A, López-Gullón JM et al (2014) Validity of hydration non-invasive indices during the weightcutting and official weigh-in for Olympic combat sports. PLoS One 9:e95336. doi:10.1371/journal.pone.0095336.g002

36. Oppliger RA, Bartok C (2002) Hydration testing of athletes. Sports Med 32:959-971

37. Armstrong LE, Soto JA, Hacker FT et al (1998) Urinary indices during dehydration, exercise, and rehydration. Int J Sport Nutr $8: 345-355$

38. Wakefield B, Mentes J, Diggelmann L, Culp K (2002) Monitoring hydration status in elderly veterans. West $\mathrm{J}$ Nurs Res 24:132-142

39. Mentes JC, Wakefield B, Culp K (2006) Use of a urine color chart to monitor hydration status in nursing home residents. Biol Res Nurs 7:197-203. doi:10.1177/1099800405281607

40. Kavouras SA, Johnson EC, Bougatsas D et al (2015) Validation of a urine color scale for assessment of urine osmolality in healthy children. Eur J Nutr. doi:10.1007/s00394-015-0905-2

41. Drabkin DL (1927) The normal pigment of the urine I. The relationship of urinary pigment output to diet and metabolism. J Biol Chem 75:443-479

42. Drabkin DL (1927) the normal pigment of the urine Ii. The relationship of the basal metabolism to the output of the normal urinary pigment. J Biol Chem 75:481-487

43. Sands JM, Layton HE (2009) The physiology of urinary concentration: an update. Semin Nephrol 29:178-195. doi:10.1016/j. semnephrol.2009.03.008

44. Hofmeyr GJ, Gülmezoglu AM, Novikova N (2002) Maternal hydration for increasing amniotic fluid volume in oligohydramnios and normal amniotic fluid volume. Cochrane Database Syst Rev. doi:10.1002/14651858.CD000134

45. Battaglia F, Prystowsky H, Smisson C et al (1960) Fetal blood studies. XIII. The effect of the administration of fluids intravenously to mothers upon the concentrations of water and electrolytes in plasma of human fetuses. Pediatrics 25:2-10

46. Santillan MK, Santillan DA, Scroggins SM et al (2014) Vasopressin in preeclampsia a novel very early human pregnancy biomarker and clinically relevant mouse model. Hypertension 64:852-859. doi:10.1161/HYPERTENSIONAHA.114.03848/-/ $\mathrm{DC} 1$ 